\title{
BURNING PLASMA PHYSICS ISSUES ILLUSTRATED BY SIMULATIONS OF FIRE
}

\author{
W. A. Houlberg \\ Oak Ridge National Laboratory \\ Oak Ridge, Tennessee 37831-8071
}

(865) $574-1350$

\begin{abstract}
Plasma startup and operating issues are examined for a burning plasma using WHIST [1] simulations of the Fusion Ignition Research Experiment (FIRE) design [2]. Fueling and density profile control issues are illustrated through pellet launch from the inboard side of the plasma. Auxiliary fast wave ion cyclotron heating and current drive are used to identify plasma startup and burn control issues. It is shown that the current rampup and bootstrap current strongly influence the evolution of the safety factor, and can be tailored to produce sawtooth-free operation for about 20 seconds of burn in well-confined high confinement mode ( $\mathrm{H}-$ mode) plasmas. Furthermore, it is also possible to maintain a reversed magnetic configuration that could allow access to the improved core confinement seen in present experiments. Access to H-mode operation is influenced by the fueling, heating and current startup waveforms. Maintenance of $\mathrm{H}$-mode conditions may require either hysterisis in the high to low confinement (H-L) back-transition or continued auxiliary heating if the fusion power is insufficient.
\end{abstract}

\section{INTRODUCTION}

Plasma transport codes that integrate the evolving geometry [2-D magnetohydrodynamic (MHD) equilibria for axisymmetric plasmas] with transport relative to magnetic flux surfaces (1-D transport) are indispensable $1-1 / 2$-D tools for planning fusion plasma experiments. This is especially true of burning plasma experiments where new or modified plasma dynamics is expected. The new dynamics is governed by the internal generation of energetic charged fusion products, the plasma heating they provide, the influence of their non-Maxwellian distribution on kinetic and magnetic characteristics, etc. In addition to these effects, many other phenomena are pushed into new regimes of plasma scale: microturbulence-induced confinement losses, density limits, internal and edge (H-mode) barrier formation and maintenance, and managing the di- vertor heat load and impurities. The $1-1 / 2-\mathrm{D}$ transport simulations identify attractive operating regimes and the means of accessing these regimes, while avoiding potential hurdles associated with density and beta limits.

In Section II we present the plasma models used in the WHIST simulations and discuss applications of these to the FIRE design in Section III.

\section{PLASMA MODELS}

The plasma particle and energy confinement is assumed to be comprised of additive contributions from neoclassical and turbulence (i.e., anomalous) processes:

$$
\begin{aligned}
\Gamma_{j}(\rho) & =\Gamma_{j}^{\mathrm{nc}}(\rho)+\Gamma_{j}^{\mathrm{an}}(\rho) \\
Q_{j}(\rho) & =Q_{j}^{\mathrm{nc}}(\rho)+Q_{j}^{\mathrm{an}}(\rho) \\
\Gamma_{j}^{\mathrm{an}}(\rho) & =-\frac{D_{j}^{\mathrm{an}}(\rho)}{a_{0}} \frac{\partial n_{j}(\rho)}{\partial \rho} \\
Q_{j}^{\mathrm{an}}(\rho) & =-\frac{n_{j}(\rho) \chi_{j}^{\mathrm{an}}(\rho)}{a_{0}} \frac{\partial T_{j}(\rho)}{\partial \rho} \\
\chi_{j}^{\mathrm{an}}(\rho) & =2 D_{j}^{\mathrm{an}}(\rho)=\chi^{\mathrm{an}}(0)\left(1+4 \rho^{2}\right)
\end{aligned}
$$

where $\rho=\sqrt{\Phi / \Phi_{\text {tot }}}$ is the flux surface label, $\Phi$ is the toroidal magnetic flux enclosed with a given flux surface and $\Phi_{\text {tot }}$ is the total toroidal flux in the plasma, $a_{0}$ is the minor radius in the plasma midplane, $\Gamma_{j}$ is the particle flux of species $j$ and $Q_{j}$ is the corresponding conduction heat flux, $n_{j}$ and $T_{j}$ are the species densities and temperatures, and $D_{j}^{\text {an }}$ and $\chi_{j}^{\text {an }}$ are the anomalous particle and heat diffusivities that are assumed to be species independent in this simplified model. The anomalous fluxes are represented by a diagonal matrix of transport coefficients in their relationship to the gradients, while a full matrix is used for the neoclassical transport (e.g., transport of helium ash has a strong component driven by the density gradient of the hydrogenic fuel ions). The anomalous transport coefficient, $\chi^{\text {an }}(0)$, is normalized to yield global low confinement mode (L-mode) according to the ITER-97L scaling [3]: 


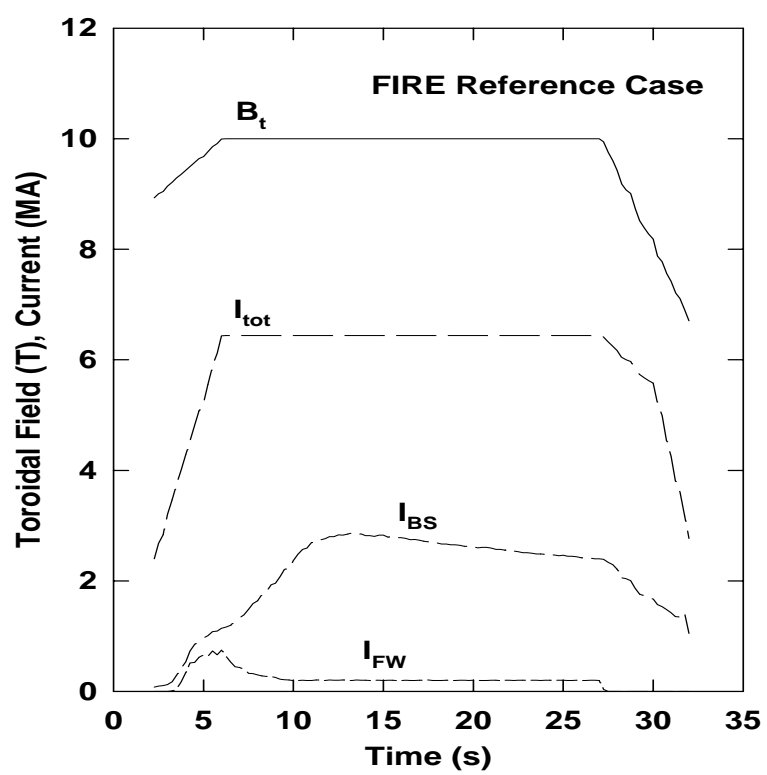

Fig. 1. Waveforms of the vacuum toroidal field, $B_{\mathrm{t}}$, total toroidal current, $I_{\text {tot }}$, fast wave current, $I_{\mathrm{FW}}$, and bootstrap current, $I_{\mathrm{BS}}$, for the reference FIRE case that transitions to the H-mode.

$$
\tau_{E}=0.023 \frac{I^{0.96} B_{t}^{0.03} n_{19}^{0.40} M^{0.2} R_{0}^{0.83} \kappa^{0.64}}{P^{0.73} \epsilon^{0.06}}(\mathrm{~s})
$$

where $I(\mathrm{MA})$ is the toroidal plasma current, $B_{t}(\mathrm{~T})$ is the toroidal magnetic field, $n_{19}$ is the plasma electron density normalized to $10^{19} \mathrm{~m}^{-3}, M$ is the average ion mass in atomic mass units, $R_{0}(\mathrm{~m})$ is the major radius, $\kappa$ is the plasma elongation, $\epsilon=a_{0} / R_{0}$ is the inverse aspect ratio, and $P(\mathrm{MW})$ is the total heating power. While this 'locally applied global model' for the anomalous transport provides a basis for scoping plasma behavior, the actual plasma response will likely be more complex because of dependencies on local gradients, differences between electron and ion transport, etc.

The high confinement mode (H-mode) is modeled by reducing the transport coefficients in the edge region in order to simulate the access. The anomalous transport prperties are suppressed by a factor of five over the region $0.95<\rho<1.0$ when the power crossing the separatrix exceeds the threshold for the transition from L-mode to H-mode using the empirical model derived from an international database IPB98-4 [4]:

$$
P_{\mathrm{thr}}=0.082 n_{20}^{0.69} B_{t}^{0.91} S^{0.96} M^{-1}(\mathrm{MW})
$$

where $n_{20}$ is the plasma electron density normalized to $10^{19} \mathrm{~m}^{-3}, S\left(\mathrm{~m}^{2}\right)$ is the plasma surface area at the separatrix, and the scaling with ion mass has been added by normalizing to results for deuterium. This algorithm typically increases the confinement by about a factor of two over L-mode. It probably does not reproduce Hmode confinement scaling - that would require a more sophisticated suppression model in which the spatial

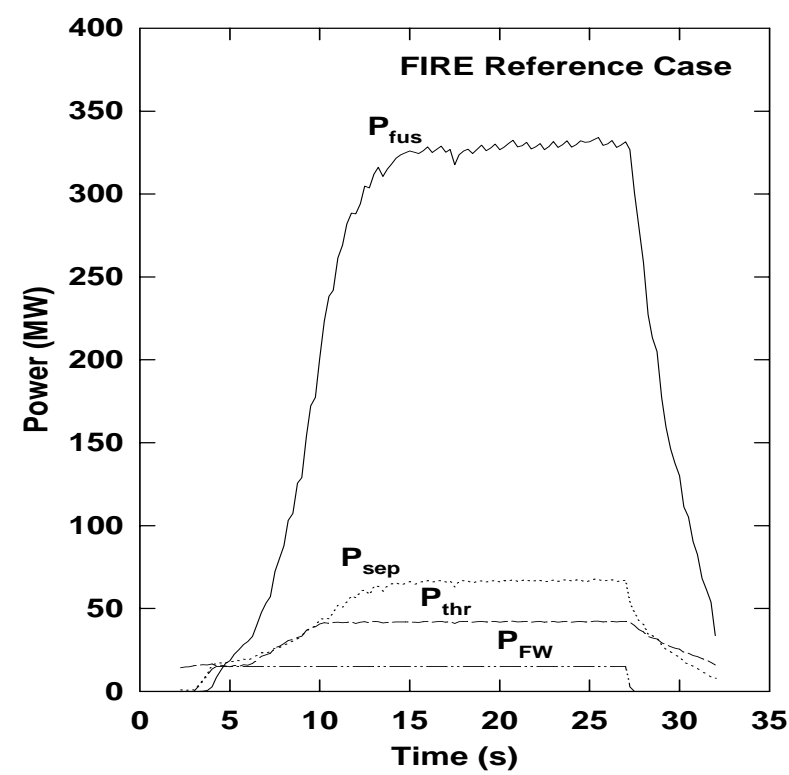

Fig. 2. The fast wave power, $P_{\mathrm{FW}}$, is ramped up during the current rise phase and held constant at $15 \mathrm{MW}$ from 4-27 s. The total fusion power, $P_{\text {fus }}$, L-H transition threshold, $P_{\mathrm{thr}}$, and power across the separatrix, $P_{\mathrm{sep}}$, are also shown.

extent and magnitude of suppression are dependent on plasma geometry and parameters.

The density perturbation from pellet injection is modeled as discrete jumps in time. Inside launch pellet injection is modeled as a uniform density perturbation that approximates the observations on DIII-D [5]. The relatively small pellets used in this study have an effective radius of $1 \mathrm{~mm}$.

An heuristic model is used for fast wave ion cyclotron range of frequency (ICRF) heating that matches weak and strong absorption limits [6]. The current drive efficiency is evaluated from the Ehst-Karney expressions [7] and is typically in the range of 0.03$0.05 \mathrm{MA} / \mathrm{MW}$ for the cases studied here. The choice of frequency and phasing was not optimized for the FIRE plasma in these simulations, so higher fast wave driven current may be feasible. The evolution of the fast alpha distribution and heating is modeled with an approximation to the time-dependent Fokker-Planck equation [8].

\section{FIRE APPLICATIONS}

The reference plasma in the FIRE design [2] is a double-null configuration with major radius $R_{0}=$ $2.0 \mathrm{~m}$, minor radius in the horizontal midplane $a_{0}=$ $0.525 \mathrm{~m}$, elongation $\kappa=1.8$, triangularity $\delta=0.4$, vacuum toroidal field $B_{t}\left(R_{0}\right)=10 \mathrm{~T}$, and toroidal current $I=6.44 \mathrm{MA}$. Time traces of the reference vacuum toroidal field and toroidal current from the WHIST simulations are shown in Fig. 1. The toroidal field 


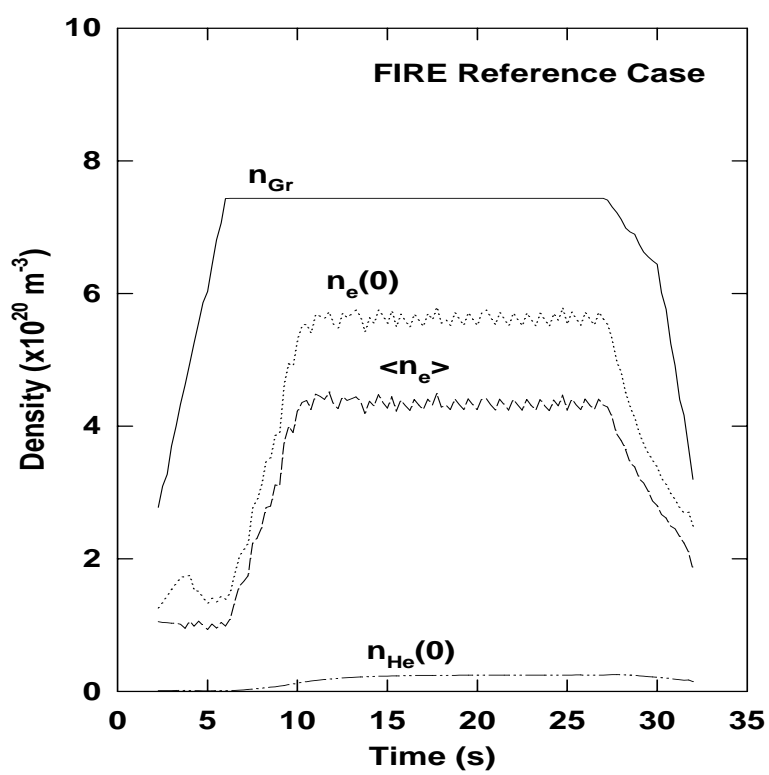

Fig. 3. The plasma density is held low during the current rise and early heating to facilitate the L-H transition. The Greenwald density limit, $n_{\mathrm{Gr}}$, axial and volume-averaged electron densities, $n_{e}(0)$ and $\left\langle n_{e}\right\rangle$, and volume-averaged helium ash density, $\left\langle n_{H e}\right\rangle$, are shown.

(TF) and current are ramped simultaneously to control formation of the current profile. This helps overcome long resistive timescale issues and compresses the startup time.

Additional traces of simulated parameters are shown in Figs 1-6 for an H-mode case in which the fast wave ICRF is initiated at $3 \mathrm{~s}$ during the current rise phase, ramped to $15 \mathrm{MW}$ between 3-4 s, and then held constant until the rampdown at $27 \mathrm{~s}$ (see Fig. 2). As seen in Fig. 1, the fast wave driven current reaches a maximum of $\approx 0.7 \mathrm{MA}$ during the current rise, but then settles down to $\approx 0.2 \mathrm{MA}$. In any case, the bootstrap current is much stronger, $I_{B S}>2 \mathrm{MA}$, over most of the burn phase, and has a dramatic effect on plasma behavior in this simulation as we will discuss further.

As shown in Fig. 2, by the time the RF power reaches its flattop value of $15 \mathrm{MW}, P_{\text {sep }}>P_{\text {thr }}$ for the L-H transition and the fusion power rises strongly in the $\mathrm{H}$ mode regime. The small oscillations in the fusion power are responses to the fuel pellets, which are injected in a feedback mode at an average rate of $\approx 4.4 \mathrm{~Hz}$ over the simulation. Each pellet contains $2.6 \times 10^{20}$ atoms in a 50-50 DT mixture and represents about $3 \%$ of the plasma mass during the flattop phase.

The fusion power of $\approx 330$ MW during the flattop is relatively high in this $\mathrm{H}$-mode case. If confinement is this good, the density or the auxiliary power can be reduced during the burn. The density is held low during the startup phase to facilitate the transition to $\mathrm{H}$-mode, and in the flat top phase the density is well below the empirical Greenwald density limit [4], as seen

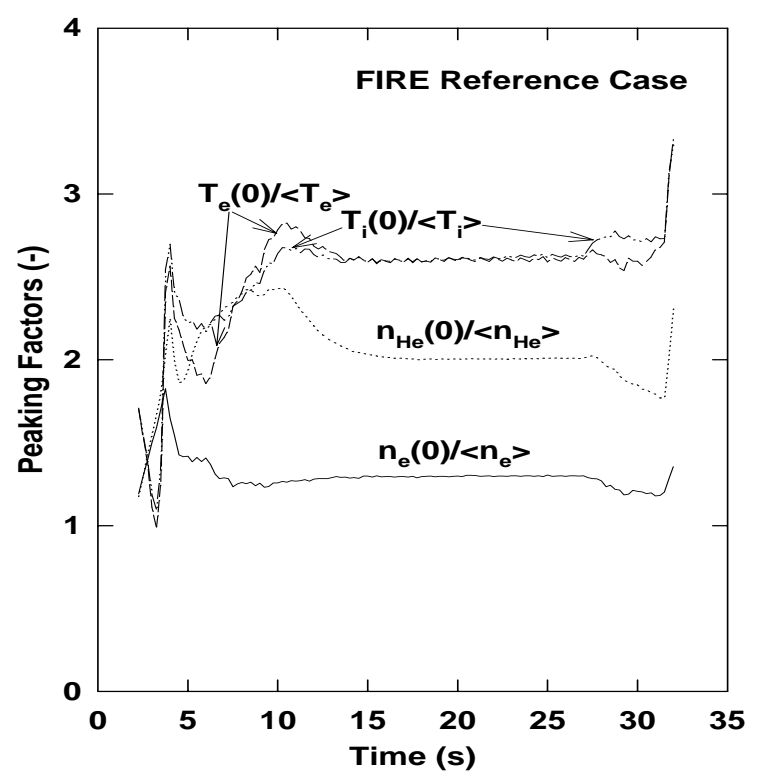

Fig. 4. The plasma profile peaking factors (axial divided by volume-averaged values) for the electron and ion temperatures, $T_{e}$, and $T_{i}$, and the electron and helium ash densities, $n_{e}$ and $n_{H e}$, show a wide variation in behavior in response to the heating and fueling during the different phases.

in Fig. 3. The helium ash density remains low, even at the axis, although $90 \%$ of the ash crossing the separatrix is recycled back to the plasma. The density oscillations are a consequence of pellet injection.

The helium ash profile is more peaked than the electron density (Fig. 4) because of the strong central peaking of the fusion source profile. Even with the assumed deep fueling from inside launch, the electron density is relatively flat. Because of the good confinement the makeup fuleing rate is low and it takes $\approx 12 \mathrm{~s}$ for pellets to replace all the fuel in the plasma. This low fueling rate is not sufficient to peak the density profile very strongly. We will show later that the density peaking is stronger in L-mode. Because of the central heating by fusion alphas, the temperature profiles are more strongly peaked than the density profile, with slight differences in the peaking factors over the course of the discharge resulting from differences in heating and transport coefficient profiles. A non-linear dependence of the transport properties on plasma density and temperature gradients (e.g., marginal stability models) could significantly modify the relationship between heating and fueling profiles and the density and temperature profiles.

The hollow bootstrap current forces the safety factor to rise on axis as seen in Fig. 5. This eventually leads to magnetic shear reversal over the inner half of the plasma beginning at $14 \mathrm{~s}$. As the current profile relaxes the reversed shear region decreases to about $30 \%$ of the minor radius. It may be possible to extend the 


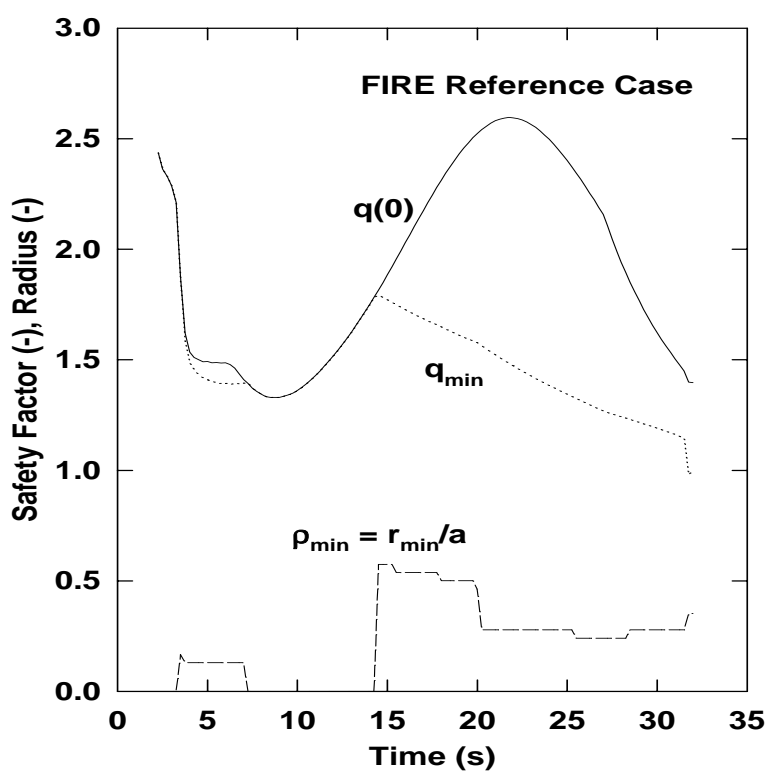

Fig. 5. The bootstrap current drives a strong shear reversal over the inner half of the plasma radius that continues to evolve during the entire burn phase. The axial and minimum safety factors, $q(0)$ and $q_{\min }$ respectively, and normalized radius of the minimum safety factor, $\rho_{\min }$, are illustrated.

reversal region with localized current drive, but this has not been examined in these simulations. Although it may be expected, there was no assumption of improved confinement or internal barrier formation in the shear reversal region of these simulations.

The fast alphas constitute $\approx 10 \%$ of the total plasma beta (Fig. 6), which is comparable to that expected in fusion reactor plasmas, and should allow examination of strong collective fast alpha phenomena. The normalized beta, $\beta_{N}=\left\langle\beta_{\text {tot }}\right\rangle(\%) a(\mathrm{~m}) B_{t}(\mathrm{~T}) / I(\mathrm{MA}) \approx 3.5$ is near the upper end of the experimental database [4].

Figure 7 illustrates the consequences of a square wave application of the full proposed auxiliary heating power of $30 \mathrm{MW}$ for FIRE. It underlines the need to tailor the startup and transition from external to internal heating in order to avoid the very high power transients that can accompany ignition, as was done in the reference case of Figs 1-6. This plasma remains ignited through the flat top phase because it is assumed to continue in $\mathrm{H}$-mode even though the power crossing the separatrix drops below the L-H transition threshold. If there no hysteresis in the back transition, the plasma would drop into L-mode shortly after termination of the auxiliary power. As in the reference case, the strong bootstrap current drives a large shear reversal region.

An L-mode case is illustrated in Figs 8-9. Although $P_{\text {sep }}$ exceeds $P_{\text {thr }}$ during the startup, the plasma is assumed to remain in L-mode. Once the flattop is reached, $P_{\text {sep }}$ is well below the threshold. Strong saw-

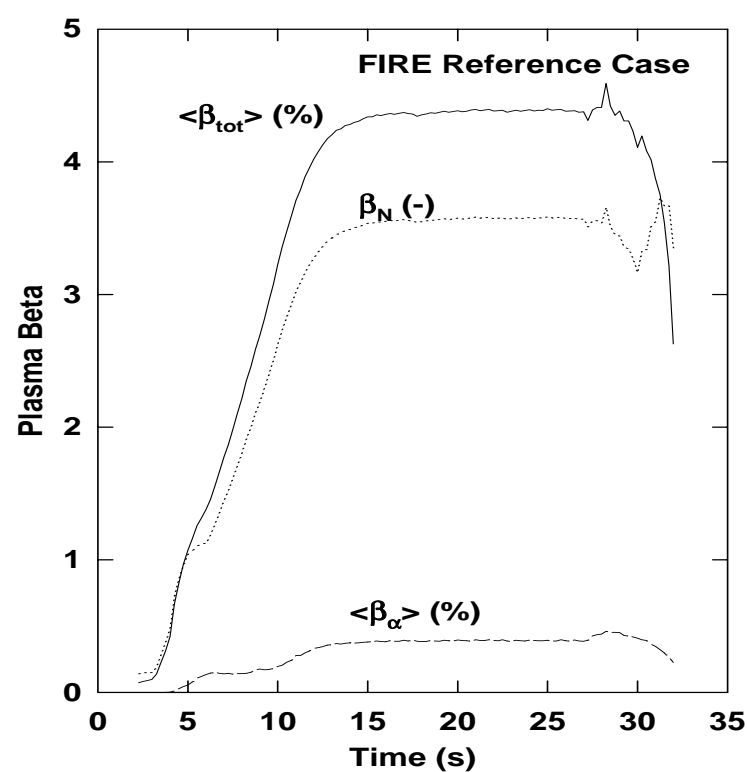

Fig. 6. The volume-averaged fast alpha pressure, $\left\langle\beta_{\alpha}\right\rangle$, is about $10 \%$ of the total volume-averaged plasma pressure, $\left\langle\beta_{\text {tot }}\right\rangle$, during the burn. Also shown is the normalized total beta, $\beta_{N}$.

tooth activity commences at $\approx 12 \mathrm{~s}$ and produces much stronger density, temperature and fusion power fluctuations than induced by the pellets. The pellet injection frequency of $6.2 \mathrm{~Hz}$ leads to stronger peaking of the density profile than the H-mode cases, even in the presence of sawtooth activity.

This L-mode case underlines the need to explore forcing the L-H transition during startup as was done in the two previous cases. Indeed, one of the primary objectives of a burning plasma device such as FIRE is to explore effective and efficient ways to make the transition from external control to self-sustainment: maximize performance while reducing the investment in auxiliary systems.

\section{ACKNOWLEDGMENTS}

This research was sponsored by Oak Ridge National Laboratory, managed by UT-Battelle for the U.S. Department of Energy under contract DE-AC0500OR22725.

\section{REFERENCES}

[1] W. A. Houlberg, S. E. Attenberger and L. M. Hively, "Contour Analysis of Fusion Reactor Plasma Performance," Nucl. Fusion, 22, 935 (1982).

[2] D. M. Meade, S. C. Jardin, J. A. Schmidt, R. J. Thome, N. R. Sauthoff, P. Heitzenroeder, B. E. Nelson, M. A. 


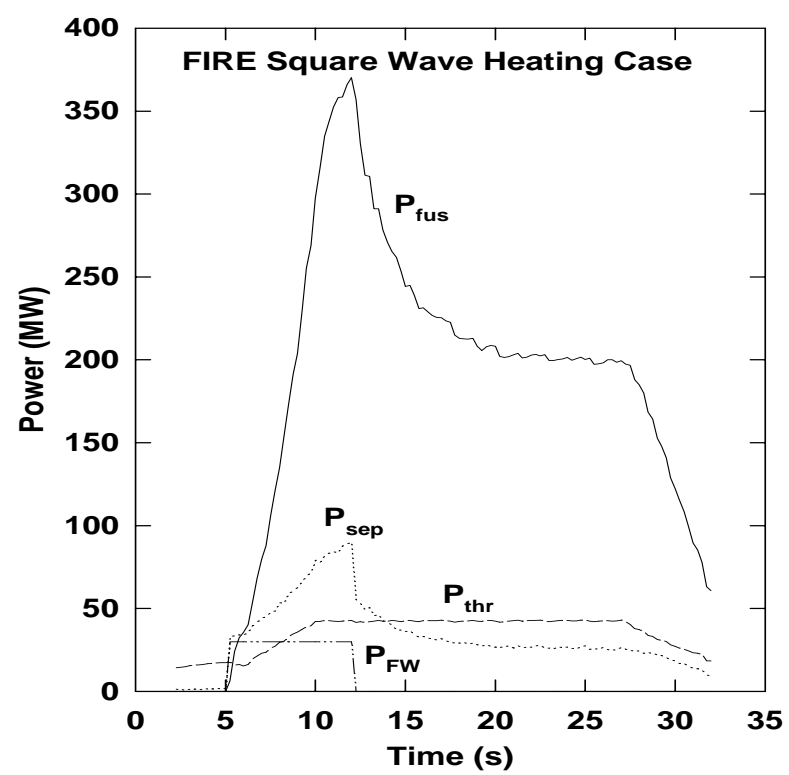

Fig. 7. A $30 \mathrm{MW}$ square application of the fast wave heating from $5-12 \mathrm{~s}$ results in a significant overshoot in the fusion power.

Ulrickson, C. E. Kessel, J. Mandrekas, C. L. Meumeyer, J. H. Schultz, P. H. Rutherford, J. C. Wesley, K. M. Young, W. M. Nevins, W. A. Houlberg, N. A. Uckan, R. W. Woolley, C. C. Baker, "Mission and Design of the Fusion Ignition Research Experiment," (Proc. 18th Int. Conf., Sorrento, 2000) IAEA, Vienna, to be published.

[3] S. M. Kaye and the ITER Confinement Database Working Group, "ITER L Mode Confinement Database," Nucl. Fusion 37, 1303 (1997).

[4] ITER Physics Expert Groups on Confinement and Transport and Confinement Modelling and Database, ITER Physics Basis Editors and ITER EDA, "Plasma Confinement and Transport," Nucl. Fusion 39, 2175 (1999).

[5] L. R. Baylor, T. C. Jernigan, P. Gohil, G. L. Schmidt, K. H. Burrell, S. K. Combs, D. R. Ernst, C. M. Greenfield, R. J. Groebner, W. A. Houlberg, C. Hsieh, M. Murakami, P. B. Parks, M. Porkolab, W. D. Sessions, G. M. Staebler, E. J. Synakowski, and the DIII-D Team, "Improved Fueling and Transport Barrier Formation with Pellet Injection from Different Locations on DIII-D," (Proc. 18th Int. Conf., Sorrento, 2000) IAEA, Vienna, to be published.

[6] W. A. Houlberg and S. E. Attenberger, "Evaluation of Current Drive Requirements and Operating Characteristics of a High Bootstrap Fraction Advanced Tokamak Reactor," Fusion Technol., 26, 566 (1994).

[7] D. A. Ehst and C. F. F. Karney, "Approximate Formula for Radiofrequency Current Drive Efficiency with Magnetic Trapping," Nucl. Fusion, 31, 1933 (1991).

[8] S. E. Attenberger and W. A. Houlberg, "Fast Alpha Diffusion and Thermalization in Tokamak Reactors," Nucl. Technol./Fusion, 4, 129 (1983).

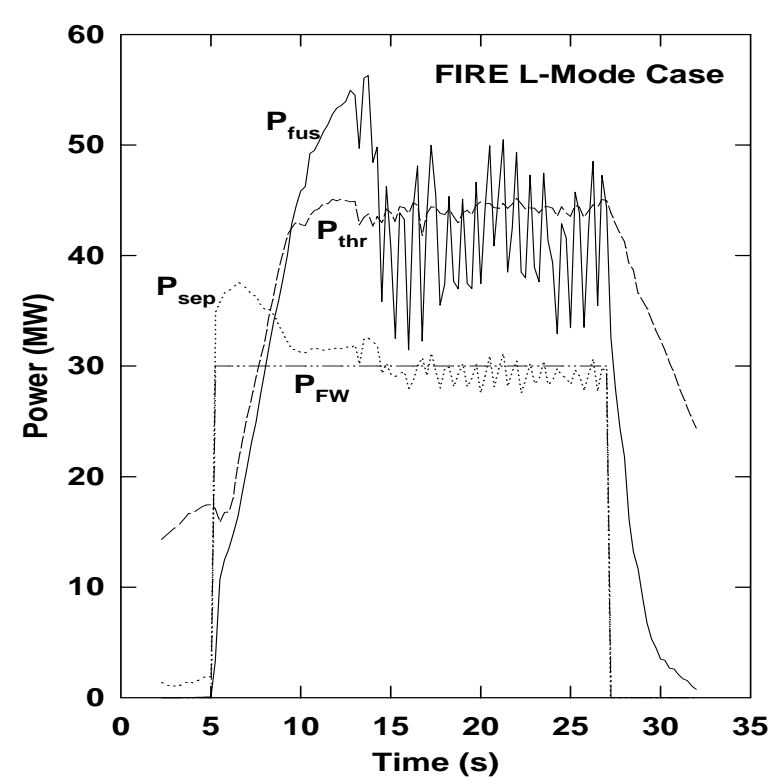

Fig. 8. Although the power crossing the separatrix exceeds the threshold for the L-H transition during the startup phase, the confinement is assumed to stay in L-Mode for this entire simulation. The amplitude of power fluctuations from sawtooth activity is much stronger than that from pellets.

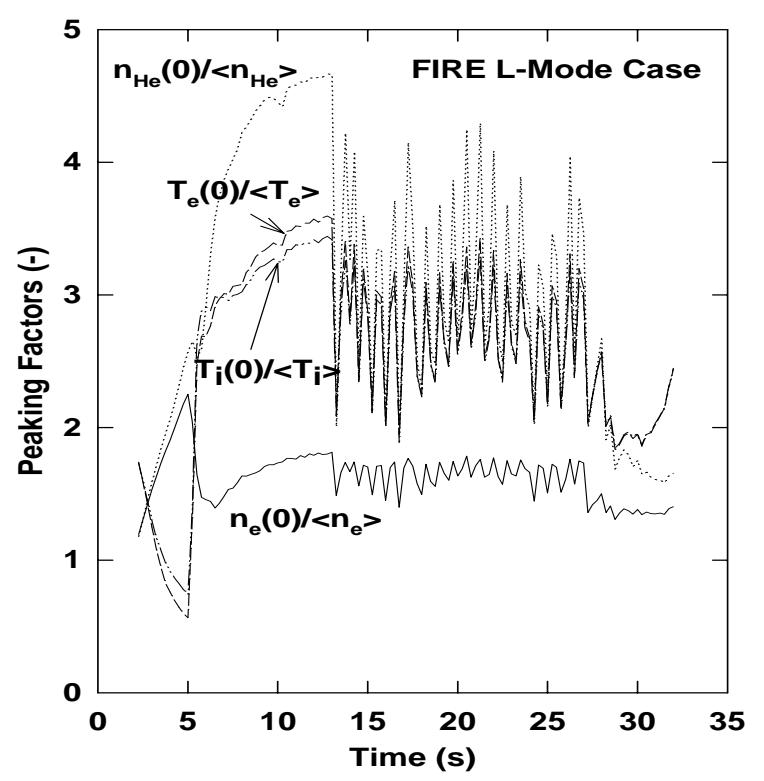

Fig. 9. Lower confinement and lower temperature operation leads to stronger density peaking even in the presence of sawtooth activity. 